BULGARIAN ACADEMY OF SCIENCES

CYBERNETICS AND INFORMATION TECHNOLOGIES • Volume 15, No 5

Special Issue on Control in Transportation Systems

Sofia • 2015

Print ISSN: 1311-9702; Online ISSN: 1314-4081

DOI: 10.1515/cait-2015-0023

\title{
Using a PicoBlaze Processor to Traffic Light Control
}

\section{Vladimir N. Ivanov}

Institute of Information and Communication Technologies, 1113 Sofia

Email:Ivanov.vladi@gmail.com

Abstract: This paper discusses the development of a complex device for Traffic Light Control. The device is based on a PicoBlaze microprocessor embedded in Spartan6 FPGA. Its benefits for the purposes of transport flows management are discussed.

Keywords: Traffic Light Control, embedded microprocessors, FPGA.

\section{Introduction}

For each country the road transport plays a significant role. It performs approximately $80 \%$ of the cargo and $75 \%$ of the passenger flow annually. The organization and regulation based on the Traffic Light Control Systems (TLCS). have played an important role for enhancing the efficiency of the traffic flows over the street-road networks in the cities. This contributes to optimization of the road transport, reducing the fuel consumption, the traffic incidents and the level of harmful gases.

There are different kinds of TLCS. They can be classified by the size, shape, number and parameters of the lights, by the technology for light generating, by the place and manner of installation, the presence or not of additional electromechanical signaling devices, types of users, to which they are related to, behaviour of the traffic lights during the day and night, behaviour in case of central power off and many other parameters. One of the most common TLCS intended for non-rail vehicles, such as motorcycles, cars, buses, trucks, etc., have three vertical 
sections. The red section is located at the top, the yellow is in the middle, and the green section is located below.

The traffic lights for pedestrians usually have only two sections: red and green. They can depict human figures or have luminous or mobile inscriptions like "Go" (Walk) and "Stop" (Do not walk). Some of the modern pedestrian traffic lights can be combined with electronic clocks showing in seconds the time remaining to switch the signal off. The walkways are also labeled by three horizontal sections with an yellow or orange light and depict the moving pedestrian crossing the road.

The trams of the public city transport have their own TLCS. They are synchronized properly with the other traffic lights, but not regulated by international agreements, and as a result, in different countries they vary considerably.

There are also mobile TLCS, with their own battery and/or photovoltaic power. They are used for temporary diversion of the traffic in cases when road works might occur. Most of the TLCS use conventional or special incandescent bulbs with power from 60 up to $100 \mathrm{~W}$. Depending on the circumstances and conditions they can be combined with reflectors, lenses, filters and hoods.

In order to achieve good performance of the light and energetic indicators, the contemporary TLCS are designed on LED lamps. They guarantee at least ten times energy saving, high brightness, smooth adjustment of its light intensity in a large range, very good focusing of the emitted light and resistance to shocks and vibrations. According to the manufacturers, the resource of 3-8 candela LED is not less than 200000 hours which means reliable operation for 20 years.

The LEDs are environmentally friendly, have high performance and small dimensions. The absence of overheating allows constructing of matrix light sources suitable for TLCS [6]. These kinds of light sources allow constant brightness perceiving at large distances that cannot be said for TLCS with a central source [9].

As a common rule, the control of the contemporary TLCS is built on the base of a microcontroller or microprocessor. A great disadvantage for most of them becomes the impossibility to be modified on real time basis. This follows from the use of pre-defined hardware and a hard written program. They have an apriori fixed timing for green, yellow and red signals, which in practice means a longer waiting time and more fuel uses by the vehicles [10].

To make the traffic light controlling more efficient, the TLCS must obtain some elements of intelligence. Such kind of TLCS forms the class of the so called Intelligent Traffic Light Control Systems (ITLCS). When ITLCS are applied for the control of traffic light signals, they usually contain a set of controllers and sensors, communication systems, adaptive control systems, traffic responsive, real-time data collection and analysis, and maintenance management systems [11]. ITLCS have the capability to share traffic signal and operational data with other systems.

The presence of an adaptive signal system is intended to improve the responsiveness of the signal timing, when the traffic conditions are rapidly changed.

The communication and sensor networks enable the use of technologies that allow the adaptive signal control to be realized. 
Their use in traffic control systems permits the achievement of greater efficiency of operation, improvement of the performance of the traffic flow systems and enables the process of automated monitoring of the equipment failures. ITLCS technology enables the process of traffic signal timing to be performed more efficiently by enhancing the data collection and system monitoring capabilities. ITLCS tools, such as automated traffic data collection, centrally controlled or monitored traffic signal systems, closed loop signal systems, interconnected traffic signals and traffic adaptive signal control, help to make the traffic signal timing process more efficient, decrease the vehicle operating costs, provide motorists with recognizable improvements in the travel time and reduce the vehicle emissions.

As pointed in [9], the use of ITLCS in Texas shows reductions of $24.6 \%$ in the delay, $9.1 \%$ in the fuel consumption, and $14.2 \%$ in the stops. In California ITLCS for Fuel Efficient Traffic Signal Management program showed reductions of $14 \%$ in the delay, $8 \%$ in the fuel consumption, $13 \%$ in the stops, and $8 \%$ in the travel time. Such a system used for improvements to an 11-intersection arterial in St. Augustine, Florida, showed reductions of $36 \%$ in the arterial delay, $49 \%$ in the arterial stops, and $10 \%$ in the travel time, resulting in an annual fuel savings of 26000 gallons.

\section{Current trends in the development of hardware based systems for managing the traffic flow}

As a rule, the traffic lights can be installed at different locations like walkways, exits of garages and parking lots of important buildings, entrances to tunnels and subways, railway crossings, etc., that require traffic control. Usually, the traffic lights are installed at intersections (Fig. 1) which can be equipped with various sensors. Thus the traffic lights can perform different functions according to the specific circumstances and situations, which require some intelligence of their management devices. For realization of ITLCS with a low level of intelligence, microcontrollers of type PIC16F877 [13] or microprocessors of type Blackfin are usually used [14].

For situations where the realization of more complex management is needed, the use of professional IBM PC computers is recommended. Overall, the use of intelligent traffic light control system capable to optimize the traffic light switching is more efficient than the conventional controller with respect to a smaller waiting time and longer distance travelled by the average vehicles. So they increase the road capacity and traffic flow, and can prevent traffic congestions.

In essence, the intelligence of a Traffic Light Controller is a kind of multifunctional device that covers a finite number of activities. As such kind of classes it can include activities related to determining the magnitude of the traffic flows with respect to individual lines of an intersection, to perform adaptive control of the light beam of traffic lights depending on the ambient light, automatic transmission of information to the higher level hierarchy to users in the traffic, and so on. 


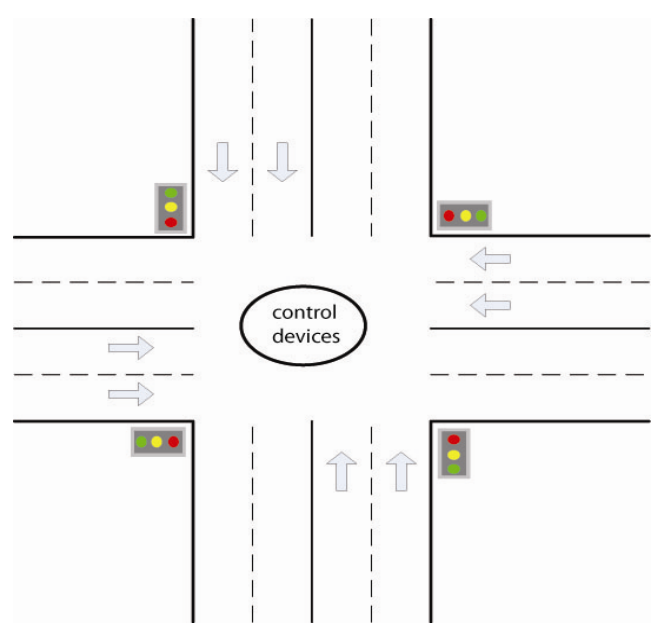

Fig. 1. Typical representation of a trivial cross road

Since these activities do not intersect themselves, and they mutually complement each other, they can be separated in different classes.

This approach allows the process of ITLCS design to be based on the created for the purposes of artificial intelligence in IPPI-RAS theory for Small Object Areas (SOA) [7]. The use of this theory ensures the transformation of the great variety of tasks in a finite set of mutually independent SOA. In this case the task of building an intelligent system for managing the traffic flows must be presented and modeled in an abstract space formed by the activities defined by each specific SOA. This means that each SOA can be realized in a single physical processor which leverages the use of the multiprocessor systems for traffic flows management. When the individual processors are realized as software generated ones, the entire ITLCS can be integrated within a single FPGA chip, which guarantees a low cost and a long period of trouble-free operation.

The purpose of this work is to present an attempt for representation of the idea for ITLCS design using the theory of SOA realized on the basis of a PicoBlaze software microprocessor , implemented in a Spartan6 FPGA structure.

Our attempt to construct the ITLCS is intended to solve the traffic situations of a trivial cross road shown in Fig. 1. It has four conventional red, yellow and green sections. It is assumed that all sensors and the associated with them devices are situated in an appropriated manner on the armature of the road intersection infrastructure. It is also assumed that ITLCS consist of intelligent devices that form the finite set of SOA capable to recognize the traffic situations and transmitters for data exchanged with other neighbouring ITLCS or with the system forming the higher level of hierarchy. Due to this, the finite set of SOA leverages the achievements of adaptive technology for traffic management based on the implementation of modern algorithms for decision making management of the traffic lights depending on the transport situation. The block diagram showing the interconnection among different SOA of the developed ITLCS is presented in Fig. 2. 


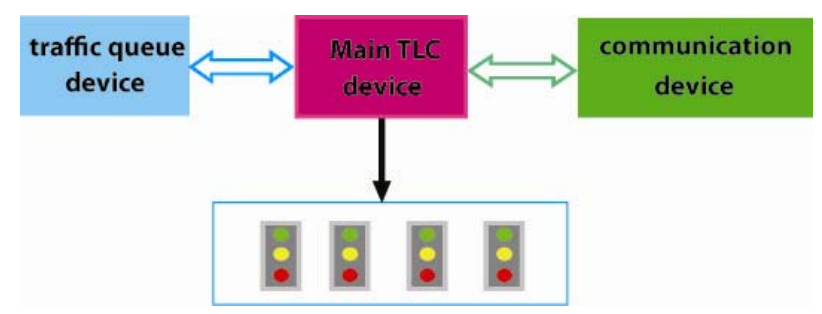

Fig. 2. Block diagram of the developed SOA based ITLCS

The aim of SOA traffic queue devices is intended to look for the traffic queue situations in 4 directions. Since the time interval of the traffic lights switching is long enough, there are no problems to recognize and to classify any traffic situations according to apriori given rules in several groups in real time.

The main role of the SOA communication device is focused to maintain the data transmission and receiving to or from other neighborhoods of ITLCS, or with the system forming a higher level of hierarchy. Here, the volume of information to be exchanged does not overcome several Kbits, the baud rate of $115 \mathrm{k}$ permits the real time mode to take also place.

The information from both SOA is fed to the main one. Based on the supplied information and in conjunction with the implemented algorithm, the main SOA makes a decision for the order and time interval of switching of the traffic lights in real time too.

As a basis for the development and physical realization of the different SOA modules, the Xilinx Spartan6 FPGA devices are used. They are realized as separated software generated embedded PicoBlaze based microprocessors, which use a common $100 \mathrm{MHz}$ clock, which allows the realization and achievement of increased functionality of the traffic flows management.

The contemporary FPGA devices contain millions of logic cells and thousands of bits of memory, which materialize their versatility [10]. They have high performance and very low power consumption; allowed parallel task processing and on the fly reconfiguration of the interior design of the system. They comply with the international standards for military and aerospace systems, which define them as an optimal element base for the development of fail-safe systems for any critical application within a single crystal [5].

In order to create the modern ITLCS, the project been decrypted is based on a high-performance software generated by 8-bit RISC PicoBlaze processor. PicoBlaze uses the internal FPGA structure in the best way. Its core is considered as one of the most successful answers of the modern trends in building the software implemented embedded processors. It requires significantly less resources compared to any other embedded microprocessors. The architecture of PicoBlaze allows full access to all hardware features of the chip. It is also possible multiple copies of the PicoBlaze architecture to be incorporated in the structure of a single FPGA device. PicoBlaze appears to be the only processor whose use dominates in the implementation of parallel algorithms within one FPGA device and the development of SoC. Due to this it can be used as a basic building block in the construction of multiprocessor systems. 
PicoBlaze was developed by $\mathrm{Ken} \mathrm{Ch}$ a p m an [2]. The structural diagram of the processor is shown in Fig. 3 [3]. It contains two banks of 8 bit general-purpose registers. The number of registers in each bank is 16 and they are fully equivalent. None of them is devoted to special purposes and does not have priority over the others. By default, the size of the program memory of the microprocessor is consistent with the structure of the FPGA block memory device and does not exceed 4k 18 bit words. PicoBlaze has a Scratchpad RAM, the size of which can take values up to 256 bytes. PicoBlaze supports up to 256 input and 256 output ports. By them it can be connected to a special interface or other logic inside or outside the FPGA. PicoBlaze is distributed as a VHDL file free. Its core requires the least resources for implementation (96 slices for mounting in Spartan-3 [4] and 26 slices for mounting in Spartan-6, Vertex, and 7 Series [3]). The performance of PicoBlaze is within 44 MIPS and over 200 MIPS when it is built in Spartan-3 and in Virtex and Seria 7 respectively. The reliability of PicoBlaze is determined by a FPGA device [5]. This permits its use as the basis for information control system development.

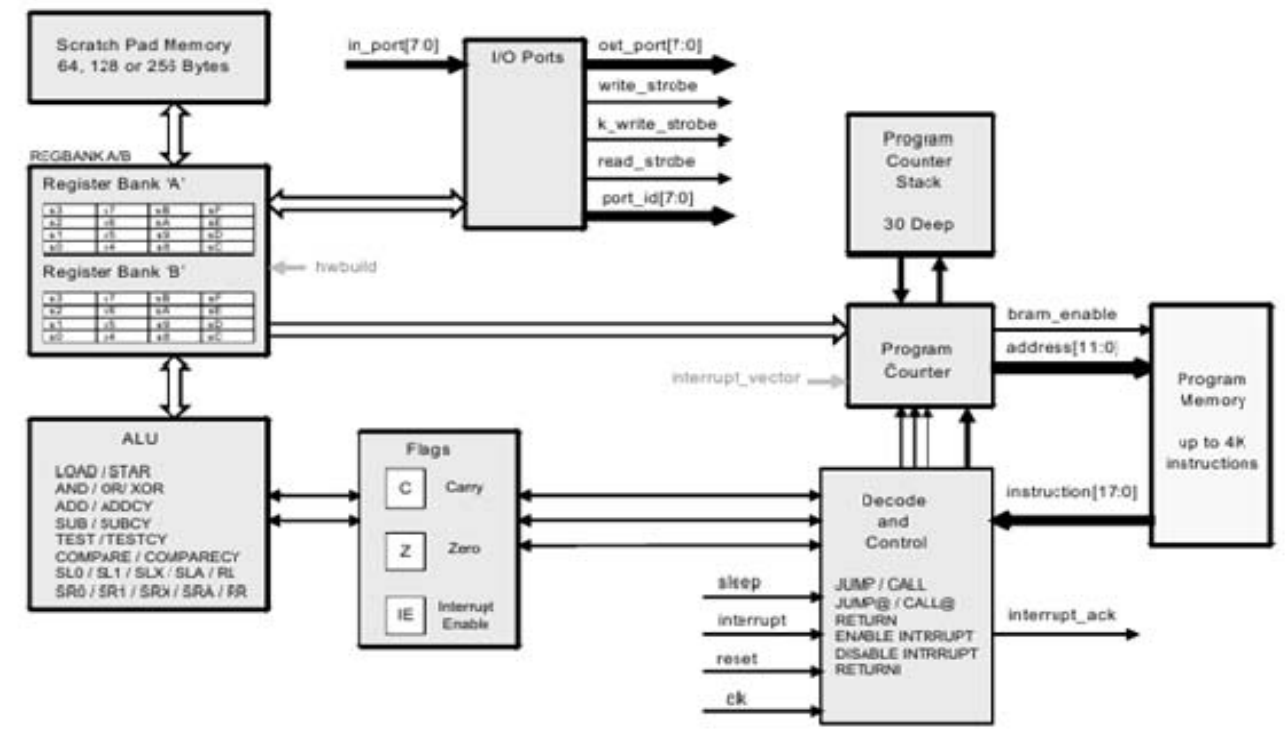

Fig. 3. Block diagram of PicoBlaze microprocessor core

\section{Structure and characteristics}

The structural diagram of the developed main TLC device is classic. It consists of a control unit, block actuators and a power supply. The control unit accepts information from the other SOA modules and forms the duration of the basic and intermediate bars of adjustment and it has the following functionalities:

- Adaptive control of the traffic stations with the possibility to adjust the duration of the light signals within the range of 1 up to $255 \mathrm{~s}$. 

interval.

- Operation of the traffic lights in a flashing mode for a selected time

- Inclusion of the street lighting in the dark hours of the day.

- Manual control of traffic lights failures of its individual sections.

- Management movement and illumination of the walkway.

- Management System horn

Ability to exchange information to and from other devices at a higher hierarchical level.

It has the ability to control up to 24 power output channels, support a maintenance calendar and time. It has the ability for direct information exchange with external PC.

The device is implemented based on FPGA device Spartan6XL4. The internal structure of the developed SOA device is shown in Fig. 4.

The structural diagram of the developed SOA that performs the duties of a communication device becomes also simple and classic. It consists of a control unit and communication bridges. It uses two classical bridges UART and SPI. They are intended to exchange information with other TLCS or with devices from higher levels of hierarchy. By default, the UART baud rate is fixed at $115 \mathrm{~Kb} / \mathrm{sec}$ but in general it can be set to any standard speed from 100 up to $900 \mathrm{~Kb} / \mathrm{sec}$. The internal structure of this SOA device is shown in Fig. 5.

Apart from the UART which becomes a hardware engine, the SPI bridge of the communication SOA based device is realized in a software manner. In case of the use of $100 \mathrm{MHz}$ system clock, the SPI Bridge offers a speed of $3.5 \mathrm{Mb} / \mathrm{s}$.

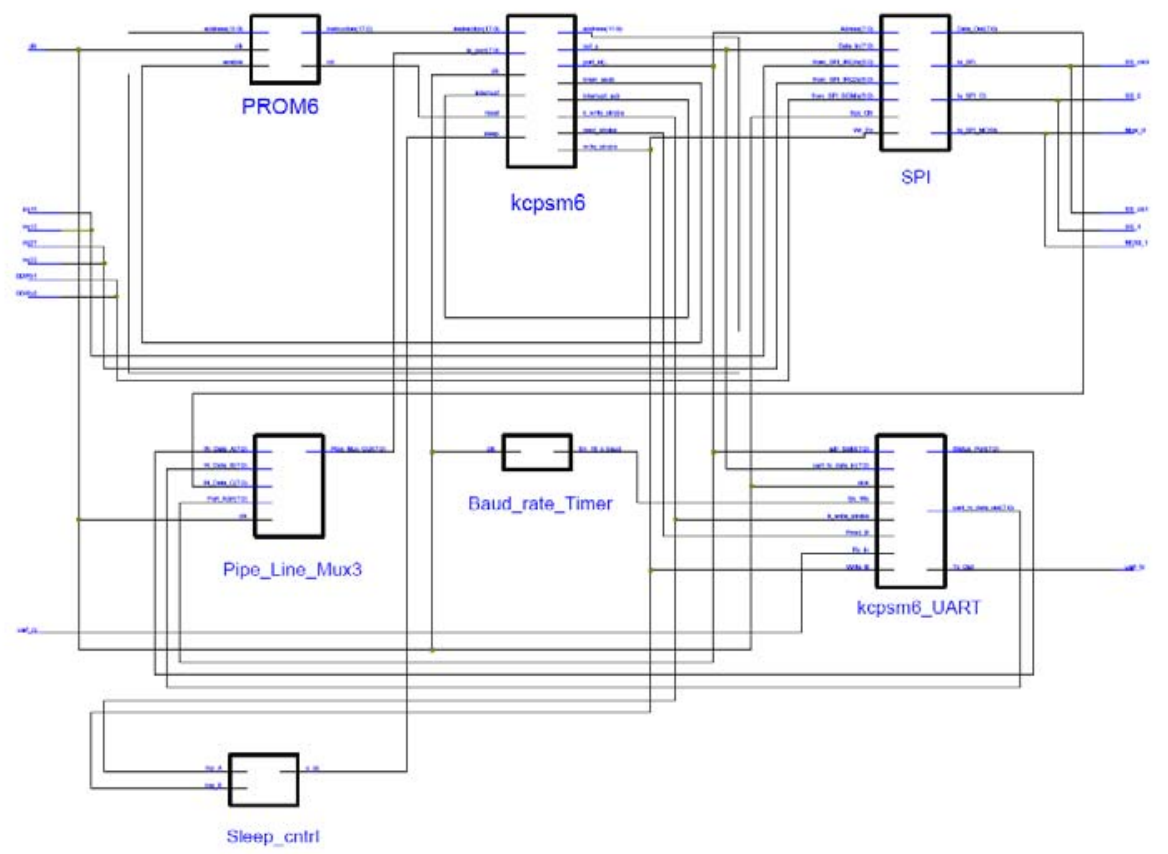

Fig. 4. Structure of the control unit 


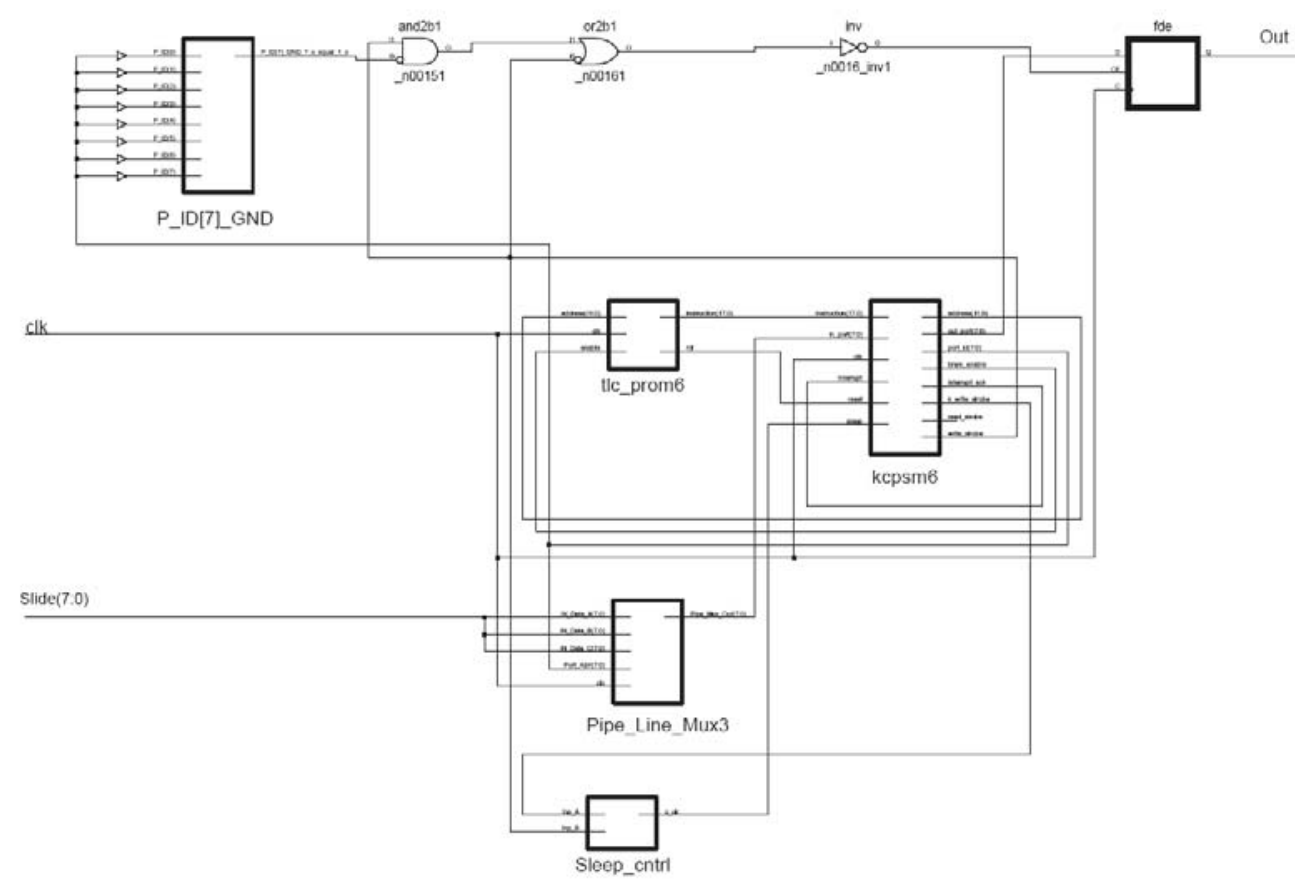

Fig. 5. Structure of the communication device

The structure of the last SOA module implemented in the design of this ITLCS intended to look for the traffic queue is also simple and depends on the algorithm used for recognition of the traffic situation. The flow of the information among different modules is realized using a register file, mapped on the input/output address map of the main SOA module.

The developed ITLCS device is designed for a 24/7 mode of work at a temperature range from 0 up to $50^{\circ} \mathrm{C}$. It is powered by $220 \mathrm{~V}$ AC main. To ensure continuous operation, an opportunity to add a battery is provided.

\section{Conclusion}

The intensity of the traffic flows in the street-road networks of cities is constantly amended, which requires their operational management through traffic and information systems. In this aspect, the developed device management traffic flows with increased functionality based on an embedded PicoBlaze microprocessor helps to improve the conditions for movement of the vehicles, decrease of the fuel consumption and the level of harmful gases. Device capabilities to manage the traffic conditions on walkways minimize the probability for accidents occurring on them. Its implementation within a FPGA device allows realizing of parallel control algorithms. The use of embedded processors opens the way for information exchange in various modes of transport and the formation of automated traffic management in road and rail transport. 
Acknowledgements: The paper is partially supported by FP7 Project 316087 ACOMIN-“Advance Computing and Innovation” and COST TU1102.

\section{References}

1. Mandloi, A., et al. High Level Modelling of Realtime Traffic Light Controller. International Journal of Engineering Science and Technology (IJEST), Vol. 4, December 2012, No 12.

2. C h a p m a n, K. PicoBlaze 8-Bit Microcontroller for Virtex Devices. XAPP213, 2002.

3. C h a p m a n, K. KCPSM6 Relies 7. www.xilinx.com

4. C h a p m a n, K. KCPSM3 for Spartan 3. Reference Design Xilinx Ltd, October 2003.

5. Device Reliability Report UG116, November 2013. www.xilinx.com

6. L u i s o v, A.V. Eye and Light. Energoatomizdat, Leningrad, 1983 (In Russian).

7. F a i n, V. S. Pattern Recognition and Machine Understanding of Natural Language. Moscow, Science, 1987 (In Russian).

8. S r in i va s, K. Implementation of FPGA in the Design of Embedded Systems Highway Traffic Light Controller. National Institute of Technology, Rourkela, May 2007.

9. Physical Encyclopedia in 5 Volumes. Moscow, Soviet Encyclopedia, 1983 (In Russian).

10. R o y e r, M., M. Goode. FPGA to ASIC Migration. - Integrated Systems Design Magazine, November, 2000.

11. S t o i l o va, K., T. S t o i l o v. Traffic Noise and Traffic Light Control. - International Journal of Transportation Research, Part D, Vol. 3, 1998, No 6, 399-417.

12. xilinx.com/t5/PicoBlaze/PicoBlaze.doc

13. www.microchip.com

14. www.analog.com 